\title{
Expression of matrix metalloproteinase-2 and mutant p53 is increased in hydatidiform mole as compared with normal placenta
}

\author{
P. PETIGNAT*†, R. LAURINI \\ *Department of Obstetrics and Gynecology, Hormone Laboratory, University of Geneva, Geneva, Switzerland; \\ tDepartment of Obstetrics and Gynecology, Gynecologic Oncology and Senology Unit, Geneva University \\ Hospitals, Geneva, Switzerland; ¥Department of Gynecology and Obstetrics, University Hospital Lund, \\ Developmental Pathology, Lund, Sweden; §Department of Gynecology and Obstetrics, University of Liege, \\ Liege, Belgium; and //Gynecologic Oncology Service, McGill University, Montreal, Canada
}

\begin{abstract}
Petignat P, Laurini R, Goffin F, Bruchim I, Bischof P. Expression of matrix metalloproteinase-2 and mutant p53 is increased in hydatidiform mole as compared with normal placenta. Int J Gynecol Cancer 2006;16:1679-1684.

Matrix metalloproteinases (MMPs) are group of enzymes thought to play an important role in trophoblastic and tumor invasion. The aim of our study was to investigate the trophoblastic expression of MMPs and p53 in normal trophoblast and hydatidiform moles (HM). Paraffin sections of 45 specimens, including 14 complete hydatidiform moles (CM), 15 partial hydatidiform moles (PM), 8 atypical partial hydatidiform moles (aPM), and 8 controls were selected. Classification of HM was established on histologic criteria and supported by the DNA ploidy results. Tissue sections from each case were immunostained with monoclonal antibodies, cytokeratin-7, MMP-2, MMP-9, tissue inhibitors of metalloproteinases (TIMP)-1, and p53 wild type (p53wt) and mutant types (mutp53). Staining for cytokeratin-7 revealed a positive reaction in 93\% of the samples. MMP-2 was mainly expressed in the syncytiotrophoblast of HM and found in $62 \%$ of aPM, 60\% PM, and 93\% CM. The mutp53 was mainly and focally expressed in syncytiotrophoblastic cells and was found in 63\% of aPM, 80\% PM, and 93\% CM. Expression of MMP-2 and mutp53 was both significantly greater in HM vs control group $(P<0.05)$ and greater in CM vs PM and aPM $(P<0.05)$. No significant difference was observed for cytokeratin-7, MMP-9, TIMP-1, and p53wt between the HM subgroups and between HM and control group. MMP-2 and mutp53 are overexpressed in HM as compared with normal trophoblast and might participate in the invasive behavior of the HM.
\end{abstract}

KEYWORDS: hydatidiform mole, MMP, p53, TIMP, trophoblastic disease.

Trophoblast invasion, like tumor invasion, is due to an active secretion of proteolytic enzymes like matrix metalloproteinases (MMPs) capable of digesting extracellular matrix ${ }^{(1)}$. Gelatinases are a group of the MMPs implicated in the invasive process by digesting collagen type IV, the major constituent of basement membrane and denatured collagen, the gelatine ${ }^{(2)}$. MMP-9 (92 kDa, gelatinase-B) and MMP-2 (72 kDa, gelatinaseA) have been shown to mediate invasion of trophoblastic cells in endometrium and proximal myometrium in

Address correspondence and reprint requests to: Patrick Petignat, MD, CHUM - Notre-Dame, 1560, Sherbrooke Est., Montréal H2L 4M1, QC, Canada. Email: patrick.petignat.chum@ssss.gouv.qc.ca

doi:10.1111/j.1525-1438.2006.00643.x normal first trimester pregnancy ${ }^{(2-4)}$. In normal trophoblastic tissue, the regulation of trophoblast invasion by MMPs is believed to be counterregulated by tissue inhibitors of metalloproteinases (TIMPs), which are secreted locally in the extracellular space where they control the activity of MMPs ${ }^{(5)}$. Among this group of enzymes, TIMP-1 is the most widely expressed and acts by inhibiting the active form of all MMPs ${ }^{(6)}$.

Hydatidiform mole (HM) refers to a pregnancyrelated disease and includes partial hydatidiform mole (PM) and complete hydatidiform mole $(\mathrm{CM})^{(7-9)}$. Unlike normal human pregnancy where trophoblast cells proliferate and differentiate to form the placenta in a regulated manner, HM placenta are overgrown and show varying degrees of local invasion and 
metastasis ${ }^{(10)}$. In CM, the villi are cystic and embryonic development is usually absent, whereas in PM, embryonic development is usually observed and a range of normal and abnormal cystic villi are present. CM have a more invasive behavior than PM and carry a greater risk of developing persistent trophoblastic tumor or metastasis ${ }^{(10-13)}$. Following the previous reports of correlation of MMPs expression with the invasiveness of tumor cells, it can be presumed that MMPs expression could be higher in HM than in normal placental tissue.

The aim of our study was to determine the immunohistochemical (IHC) expression of MMP-2, MMP-9, TIMP-1, and p53 in normal trophoblast compared with $\mathrm{HM}$ and its subgroups. p53 was chosen because this oncosuppressor is a potent regulator of $\mathrm{MMPs}^{(14)}$.

\section{Materials and methods}

\section{Patients}

Cases with HM $(n=45)$ were collected from the files of the Pathology Department, University Hospital of Lausanne, Switzerland. Histologic evaluation of all cases was performed on routine sections stained with hematoxylin and eosin using published criteria by Szulman and Surti ${ }^{(8)}$. The slides were evaluated by expert pathologist (R.L.) with special expertise in trophoblastic disease. As controls, six cases were collected from clinically normal pregnancies interrupted by vacuum for psychosocial or medical reasons that were unlikely to affect placental structure and function, and two cases were collected after surgical curettage because of incomplete spontaneous abortion without hydropic changes.

\section{Histopathology}

CM and PM were classified according to histologic criteria and DNA ploidy results. Cases that did not fulfill the conventional morphologic criteria for either $\mathrm{CM}$ or PM were classified as atypical PM (aPM). This included specimens where on gross examination there were no vesicular changes (hydropic villi with diameter of $5 \mathrm{~mm}$ or over) as can be seen in both complete (all vesicular) and partial (focal vesicular) moles (Fig. 1). Histology of these aPM shows diffuse hydropic villi (up to $2-3 \mathrm{~mm}$ in diameter) changes characterized by a focal branching hyperplasia of the syncytiotrophoblast (ST) without bilaminar (syncytio and cytotrophoblast) and circumpherential trophoblast proliferation, as observed in complete and partial moles. A total of eight such cases were diagnosed as aPM, 15 as PM, and 14 as CM.

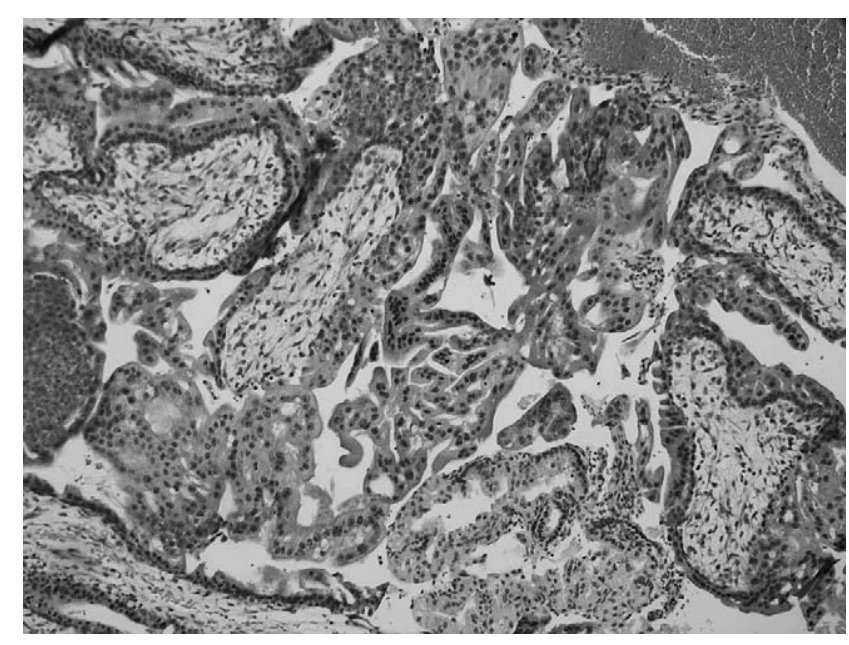

Figure 1. Atypical molar pregnancy with chorionic villi presenting a prominent ST showing a branching pattern. H\&E $260 \times$.

\section{DNA analysis}

DNA analysis was performed with the technique described previously by Hedley et $a l^{(15)}$. This was performed in most of the cases on the same paraffin block that was used for hematoxylin and eosin staining. The samples mainly presented chorionic villi with limited contamination from membranes and deciduas. A placenta was classified as tetraploid if the peak in the G2/M region appeared in more than $25 \%$ of the cells and the DNA index was between 1.90 and 2.1 and triploid if the DNA index was between 1.4 and 1.60. Almost all cases (13/14) histologically diagnosed as $\mathrm{CM}$ yielded diploid histograms by flow cytometry, whereas 6 of the 8 histologically classified as aPM and 11 of 15 diagnosed as PM yielded a triploid histogram (Table 1).

\section{Immunohistochemistry}

IHC studies were performed on formalin-fixed, paraffin-embedded tissue section using the avidinbiotin-peroxidase complex method ${ }^{(16)}$. Sections were cut $(5 \mu \mathrm{m})$ thick, deparaffinized in xylene, and dehydrated in a descending ethanol series (from $100 \%$ to $70 \%$ ). The antibody for cytokeratin-7 was mouse monoclonal antibody anti-human cytokeratin-7 (Dako, Glostrup, Denmark; diluted 1:500). The antibodies for MMP-2 and MMP-9 were mouse monoclonal antibodies (both from Oncogene Research, Steheten AG, Basel, Switzerland) and used at a 1:400 dilution. The antibody to TIMP-1 was a polyclonal (Ab-1) from Anawa (Wanger, Switzerland) and was used at a dilution of 1:100. Antibody for p53 mutated (mutp53) (Ab3 ) and P53 wild type (p53wt) (Ab-5) were both from 
Table 1. Clinical characteristics of patients with normal trophoblast, aPM, PM and CM

\begin{tabular}{lllll}
\hline & Control group $(n=8)$ & aPM $(n=8)$ & PM $(n=15)$ & CM $(n=14)$ \\
\hline Age $^{a}$ & $25(15-31)^{*}$ & $26(21-38)^{*}$ & $31(22-43)^{*}$ & $28(14-44)^{*}$ \\
GA & $10(9-21)^{*}$ & $12(10-14)^{*}$ & $12(7-21)^{*}$ & $11(8-13)^{*}$ \\
\hline
\end{tabular}

GA, gestational age (GA unknown for two CM cases).

* Results are shown as median (range).

${ }^{a}$ Maternal age at diagnosis.

Oncogene Research and used at a 1:10 dilution. A section of breast cancer with a known negative immunoreactivity for p53wt and positive for mutp53 was included as a positive control.

\section{Quantitative evaluation of cytokeratin-7, MMP-2, MMP-9, TIMP-1, p53wt, and mutp53}

Immunoreactivity of the tissue was graded semiquantitatively according to the intensity of staining as negative $(-)$, weakly positive $(<50 \%$ positive $=+)$, and strongly positive $(>50 \%$ positive $=++)$. Cellular localizations and the frequency of positive reactions for each antigen were recorded. For all cases, the presence or absence of nuclear staining was assessed in the three following cell types: cytotrophoblast, ST, and extravillous trophoblast (EVT). All the evaluation was performed by the expert pathologist blinded to the original diagnosis, the hematoxylin and eosin histologic appearance, and to the results of cytometry. Hofbauer cells were not considered because their positivity can be the result of macrophagic activity.

\section{Statistical analysis}

Statistical analysis was performed using the Stata Statistic software package (release 5.0; Stata Corporation, College Station, TX). The correlation between categorical variables was assayed by using the two-tailed Fisher exact test. All $P$ values resulted from the use of two-sided statistical tests and $P$ values $<0.05$ indicated statistically significant differences.

\section{Results}

\section{Patient's characteristics}

The median ages for the patients at diagnosis and gestational age for the different histology are given in Table 1. All original diagnoses were based on morphology and DNA ploidy analysis (Table 2), as reported in the Materials and methods section.

\section{Immunohistrochemistry}

Staining for cytokeratin-7 revealed a positive reaction (cytotrophoblast) in 42 out of 45 samples, cytokeratinpositive samples were cases as well as controls, and no significant differences could be seen between the groups (Fig. 2).

p53wt did not show any immunoreactivity in normal trophoblast (Table 3). In the HM group, only few cases $(3 / 37)$ showed a weak expression in cytotrophoblastic cells.

Positive albeit focally expression of mutp53 was observed in most $(81 \%)$ of the HM cases. However, in the control group no expression was found, except in one case (Table 3). Positive cells were found to be restricted to the villous trophoblast, essentially in the ST, whereas EVT showed an absence of immunostaining. There was a statistical significance in mutp53 expression between normal placenta and HM $(P<0.05$, Fisher exact test).

The percentage of positive expression of mutp53 varied in different groups of HM. In CM immunoreactive nuclei were found in $93 \%$ of cases. The expression of mutp53 was significantly higher in CM with obvious hyperplasia of trophoblast.

In the other groups, mutp53 was positive in $63 \%$ of aPM and $80 \%$ of PM (Table 3) (Fig. 3). There was a statistical significance in mutp53 expression between normal placenta and HM $(P<0.05$, Fisher exact test).

MMP-2 was essentially expressed in the ST (except in the control group) and EVT in normal trophoblast and HM (Table 4) (Fig. 4). The ST in PM and CM had significantly increased positivity for MMP-2 as compared with the control group $(P<0.05)$ (Table 4$)$.

Table 2. Flow cytometry analysis of DNA ploidy in normal trophoblast, aPM, PM, and CM

\begin{tabular}{lllll}
\hline Sample & $\begin{array}{l}\text { Total } \\
N\end{array}$ & $\begin{array}{l}\text { Diploid } \\
N(\%)\end{array}$ & $\begin{array}{l}\text { Triploid } \\
n(\%)\end{array}$ & $\begin{array}{l}\text { Tetraploid } \\
n(\%)\end{array}$ \\
\hline Control group & 8 & $8(100)$ & - & - \\
aPM & 8 & - & $6(75)$ & $2(25)$ \\
PM & 15 & - & $11(73)$ & $4(27)$ \\
CM & 14 & $13(93)$ & - & $1(7)$ \\
\hline
\end{tabular}




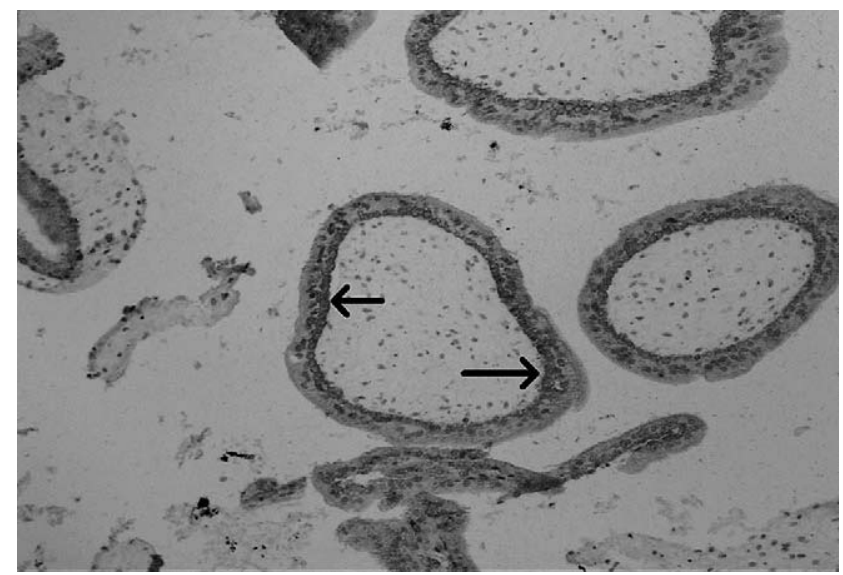

Figure 2. Strongly positive cytokeratin-7 immunoreactivity in villous cytotrophoblast (arrows) from molar pregnancy. 100×.

MMP-9 expression was observed in all the groups (control group, aPM, PM, and CM), in the cytotrophoblast, and rarely in the EVT (Table 4). No significant difference was observed in MMP-9 expression between normal placenta and HM.

TIMP-1 expression was weak in almost all specimens. We observed only a few positive cases in the ST of aPM, PM, and CM. The slightly increased expression of TIMP-1 was observed in the HM group as compared with normal pregnancy and did not reach statistical significance.

\section{Discussion}

The role of MMPs and their inhibitors in the invasive properties of trophoblastic cells has been studied in normal human placenta ${ }^{(17)}$. In cancer, MMPs have also been studied, and it seems that tumors cells utilize the matrix-degrading capability of these enzymes to spread to distant sites and to promote the growth of the cells once they have metastatized ${ }^{(18)}$. To date, limited research has been carried out on the role of MMPs in HM.

Because HM are characterized by an abnormal growth of chorionic tissues with varying degrees of local invasion and metastases, it can be assumed that MMPs could be differently expressed in this type of abnormal conception. In the present study, we tested this hypothesis by IHC analysis of MMP-2, MMP-9, TIMP-1, p53wt, and mutp53 in normal placenta, aPM, $\mathrm{PM}$, and CM.

MMP-2 expression was reported to be higher in HM than in first trimester placenta ${ }^{(19,20)}$. This is consistent with the results of the present study, where stronger staining was observed in the villous trophoblast of $\mathrm{aPM}, \mathrm{PM}$, and CM as compared with normal placenta. Moreover, we observed a significant increased expression of MMP-2 in the HM subgroups compared with the control group, with CM having an enhanced positivity as compared with aPM and PM. The fact that MMP-2 expression was observed mainly in ST and in EVT suggests that this protein can be an indicator of proliferative and invasive activity. This overexpression could contribute to the higher risk of neoplastic behavior generally observed in HM.

In our study, expression of MMP-9 was similar in all four subgroups. This is consistent with the IHC study of Vegh et al. ${ }^{(19)}$ and the quantitative studies using specific enzyme-linked immunosorbent assays performed by Okamoto et al. ${ }^{(20)}$ where no significant difference in MMP-9 levels were observed between first trimester placenta and HM.

Expression of TIMP-1 was found in ST in all four subgroups, but in general, staining was weak. There was no significant difference between normal placenta and HM. Okamoto et al. ${ }^{(20)}$ observed also that TIMP-1 expression was similar between normal and molar pregnancy. In contrast, these investigators observed that TIMP-2 was significantly reduced at both mRNA and protein levels in the HM tissue (5.8-fold decrease), and they suggested that the imbalance of MMP-2/ TIMP-2 in favor of MMP-2 activity may be a critical factor for trophoblast invasion ${ }^{(20)}$.

The tumor-suppressor gene p53 is known to play a central role in the protection against the propagation of DNA damage, primarily by inducing cell cycle

Table 3. Mutant p53expression in normal trophoblast, CM, PM, and aPM

\begin{tabular}{|c|c|c|c|c|c|c|c|c|c|c|c|c|c|}
\hline & & \multicolumn{3}{|c|}{ Control group $(n=8)$} & \multicolumn{3}{|c|}{$\mathrm{aPM}(n=8)$} & \multicolumn{3}{|c|}{$\mathrm{PM}(n=15)$} & \multicolumn{3}{|c|}{$\mathrm{CM}(n=14)$} \\
\hline & & $-(\%)$ & $+(\%)$ & $++(\%)$ & $-(\%)$ & $+(\%)$ & $++(\%)$ & $-(\%)$ & $+(\%)$ & $++(\%)$ & $-(\%)$ & $+(\%)$ & $++(\%)$ \\
\hline \multirow[t]{3}{*}{ p53 mutant type } & ST & $7(88)$ & $1(12)$ & - & $3(37)$ & $5(63)$ & - & $3(20)$ & $12(80)$ & - & $1(7)$ & $10(72)$ & $3(21)$ \\
\hline & $\mathrm{CT}$ & $8(100)$ & - & - & $6(75)$ & $2(25)$ & - & $15(100)$ & - & - & $14(100)$ & - & - \\
\hline & EVT & $8(100)$ & - & - & $8(100)$ & - & - & $15(100)$ & - & - & $14(100)$ & - & - \\
\hline \multirow[t]{3}{*}{ p53 wild type } & ST & $8(100)$ & - & - & $8(100)$ & - & - & $15(100)$ & - & - & $14(100)$ & - & - \\
\hline & $\mathrm{CT}$ & $8(100)$ & - & - & $8(100)$ & - & - & $14(93)$ & $1(7)$ & - & $12(79)$ & $2(21)$ & - \\
\hline & EVT & $8(100)$ & - & - & $8(100)$ & - & - & $15(100)$ & - & - & $14(100)$ & - & - \\
\hline
\end{tabular}

CT, cytotrophoblast; EVT, extravillous trophoblast; ST, syncytiotrophoblast. 


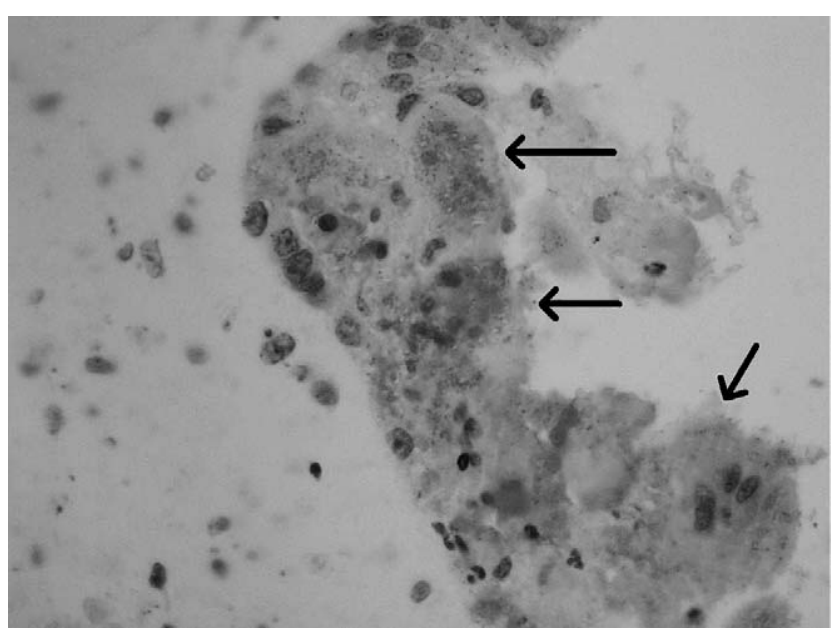

Figure 3. Distinctly positive MMP-2 immunoreactivity in proliferating syncytium trophoblast (arrows) from molar pregnancy. $100 \times$.

arrest or apoptosis. Mutation of p53, which is the most frequent genetic alteration detected in human cancer, inactivates this growth regulatory function and causes a loss of tumor-suppressive activity ${ }^{(21)}$. In most of the studies, p53 expression is increased in HM, but this overexpression is presumed to be due to an upregulation of p53wt rather than the mutp53 gene ${ }^{(21-31)}$. In our study, using conventional IHC for detecting p53wt, we have found no immunoreactivity in normal pregnancy and scattered immunoreactivity in ST cells in HMs. As reported previously, p53wt may be difficult to demonstrate in trophoblastic tissue. Reasons may be the short half-life of the p53wt protein, the conformational state of the p53 protein, which is not recognized by the antibody p53wt, or the p53 binds to cytoplasmic or nuclear proteins and the epitodes are not recognized by the conformation-specific monoclonal antibodies ${ }^{(32)}$. It is generally considered that the increased expression observed of p53 in normal pla-

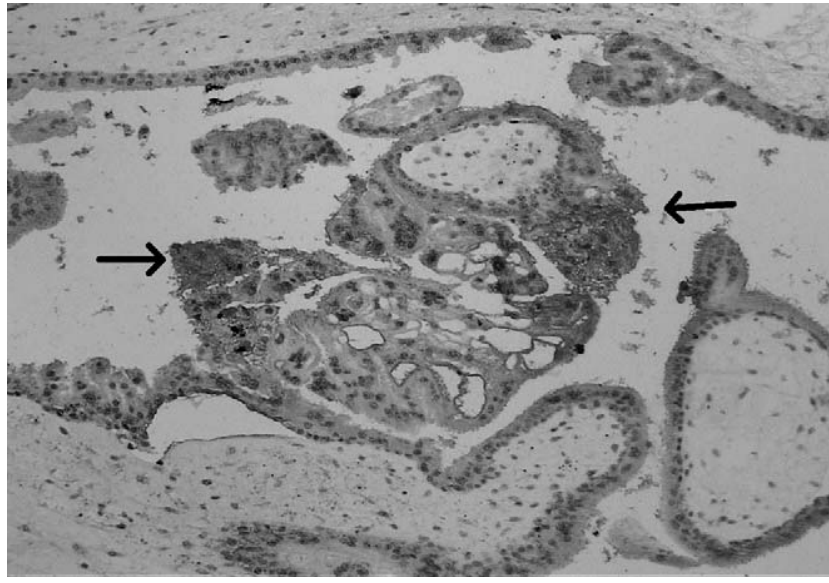

Figure 4. Focally positive p53 mutant immunoreactivity in ST (arrows) from molar pregnancy. $100 \times$.

centa is not due to a mutation of the gene as in malignant tumors but rather to a physiologic upregulation that could be a mechanism for controlling trophoblast proliferation and invasion in normal placenta ${ }^{(33)}$. Our results indicate that expression of mutp53 is significantly increased in $\mathrm{HM}$ as compared with control group, but these findings do not necessarily mean that the mutp53 has lost its suppressive activity. It has been suggested previously that different mutations do not essentially lead to a completely identical phenotype and that only some specific mutations may contribute to a more aggressive behavior ${ }^{(34)}$. Webley et al. ${ }^{(34)}$ proposed that the various mutp53 should be classified according to their conformation as recognized by antibodies. Thus, our results suggest that the mutp53 are increased in HM as compared with normal placenta, but it could be a different conformation of tertiary folding of the polypeptide or binding to proteins rather than a true gene mutation. It is possible that the different phenotype observed in HM may confer different biologic activity.

Table 4. Immunostaining of normal trophoblasts, CM, PM, and aPM

\begin{tabular}{|c|c|c|c|c|c|c|c|c|c|c|c|c|c|}
\hline & & \multicolumn{3}{|c|}{ Control group $(n=8)$} & \multicolumn{3}{|c|}{$\mathrm{aPM}(n=8)$} & \multicolumn{3}{|c|}{$\mathrm{PM}(n=15)$} & \multicolumn{3}{|c|}{$\mathrm{CM}(n=14)$} \\
\hline & & $-(\%)$ & $+(\%)$ & $++(\%)$ & $-(\%)$ & $+(\%)$ & $++(\%)$ & $-(\%)$ & $+(\%)$ & $++(\%)$ & $-(\%)$ & $+(\%)$ & $++(\%)$ \\
\hline \multirow[t]{3}{*}{ MMP-2 } & ST & $8(100)$ & - & - & $3(38)$ & $4(50)$ & $1(12)$ & $6(40)$ & $9(60)$ & - & $1(7)$ & $7(50)$ & $6(43)$ \\
\hline & CT & $8(100)$ & - & - & $8(100)$ & - & - & 15 (100) & - & - & $14(100)$ & - & - \\
\hline & EVT & $2(25)$ & $6(75)$ & - & $6(25)$ & $2(25)$ & - & $11(73)$ & $3(20)$ & $1(7)$ & $13(93)$ & $1(7)$ & - \\
\hline \multirow[t]{3}{*}{ MMP-9 } & ST & $8(100)$ & - & - & $8(100)$ & - & - & 15 (100) & - & - & $14(100)$ & - & - \\
\hline & CT & - & $8(100)$ & - & - & $1(12)$ & $7(87)$ & $3(20)$ & $8(53)$ & $4(27)$ & - & $13(93)$ & $1(7)$ \\
\hline & EVT & $8(100)$ & - & - & $8(100)$ & - & - & $13(87)$ & - & $2(13)$ & $14(100)$ & - & - \\
\hline \multirow[t]{3}{*}{ TIMP-1 } & ST & $8(100)$ & - & - & $7(88)$ & $1(12)$ & - & $13(77)$ & $2(13)$ & - & $11(88)$ & $3(22)$ & - \\
\hline & CT & $8(100)$ & - & - & $8(100)$ & - & - & 15 (100) & - & - & 14 (100) & - & - \\
\hline & EVT & $8(100)$ & - & - & $8(100)$ & - & - & 15 (100) & - & - & 14 (100) & - & - \\
\hline
\end{tabular}

CT, cytotrophoblast; EVT, extravillous trophoblast; ST, syncytiotrophoblast. 
Collectively, the present study demonstrates that MMP-2 and mutp53 are increased in HM as compared with normal placenta indicating that they may play a role in uncontrolled trophoblastic proliferation observed in HM. Finally, the various published reports are contradictory regarding mutp53; this may be partly explained by the different technologies used, the different clinical characteristics of patients, and the potential pitfalls in IHC. Further larger studies are probably needed to determine the role of mutp53 type in the pathogenesis of HM disease.

\section{Acknowledgments}

The authors thank L. Haenggeli for skillful help in immunohistochemical staining and the collaborators of Institute of Pathology of Lausanne (CHUV) for preparing the specimens.

\section{References}

1 Bischof P, Meisser A, Campana A. Paracrine and autocrine regulators of trophoblast invasion-a review. Placenta 2000;21(Suppl. A):S55-S60.

2 Bischof P, Martelli M, Campana A, Itoh Y, Ogata Y, Nagase H. Importance of matrix metalloproteinases in human trophoblast invasion. Early Pregnancy 1995;1:263-9.

3 Librach CL, Werb Z, Fitzgerald ML et al. 92-kD type IV collagenase mediates invasion of human cytotrophoblasts. J Cell Biol 1991;113:437-49.

4 Pijnenborg R, Dixon G, Robertson WB, Brosens I. Trophoblastic invasion of human decidua from 8 to 18 weeks of pregnancy. Placenta 1980;1:3-19.

5 Bischof P, Meisser A, Campana A. Mechanisms of endometrial control of trophoblast invasion. J Reprod Fertil Suppl 2000;55:65-71.

6 Goldberg GI, Strongin A, Collier IE, Genrich LT, Marmer BL. Interaction of $92-\mathrm{kDa}$ type IV collagenase with the tissue inhibitor of metalloproteinases prevents dimerization, complex formation with interstitial collagenase, and activation of the proenzyme with stromelysin. J Biol Chem 1992;267:4583-91.

7 Vassilakos P, Riotton G, Kajii T. Hydatidiform mole: two entities. A morphologic and cytogenetic study with some clinical consideration. Am J Obstet Gynecol 1977;127:167-70.

8 Szulman AE, Surti U. The syndromes of hydatidiform mole. II. Morphologic evolution of the complete and partial mole. Am J Obstet Gynecol 1978;132:20-7.

9 Szulman AE, Surti U. The syndromes of hydatidiform mole. I. Cytogenetic and morphologic correlations. Am J Obstet Gynecol 1978;131:665-71.

10 Berkowitz RS, Goldstein DP. Chorionic tumors. N Engl J Med 1996;335:1740-8.

11 Osathanondh R, Goldstein DP, Pastorfide GB. Actinomycin D as the primary agent for gestational trophoblastic disease. Cancer 1975;36:863-6.

12 Palmer JR. Advances in the epidemiology of gestational trophoblastic disease. J Reprod Med 1994;39:155-62.

13 Seckl MJ, Fisher RA, Salerno G et al. Choriocarcinoma and partial hydatidiform moles. Lancet 2000;356:36-9.
14 Meyer E, Vollmer JY, Bovey R, Stamenkovic I. Matrix metalloproteinases 9 and 10 inhibit protein kinase C-potentiated, p53mediated apoptosis. Cancer Res 2005;65:4261-72.

15 Hedley DW, Friedlander ML, Taylor IW, Rugg CA, Musgrove EA. Method for analysis of cellular DNA content of paraffin-embedded pathological material using flow cytometry. I Histochem Cytochem 1983;31:1333-5.

16 Hsu SM, Raine L, Fanger H. The use of antiavidin antibody and avidin-biotin-peroxidase complex in immunoperoxidase technics. Am J Clin Pathol 1981;75:816-21.

17 Bischof P, Irminger-Finger I. The human cytotrophoblastic cell, a mononuclear chameleon. Int J Biochem Cell Biol 2005;37:1-16.

18 Sequoia Ecosystem and Recreation Preserve Act of 1999. In 106th Congress ed.; 1999.

19 Vegh GL, Selcuk Tuncer Z, Fulop V, Genest DR, Mok SC, Berkowitz RS. Matrix metalloproteinases and their inhibitors in gestational trophoblastic diseases and normal placenta. Gynecol Oncol 1999;75: 248-53.

20 Okamoto T, Niu R, Yamada S, Osawa M. Reduced expression of tissue inhibitor of metalloproteinase (TIMP)-2 in gestational trophoblastic diseases. Mol Hum Reprod 2002;8:392-8.

21 Cadwell C, Zambetti GP. The effects of wild-type p53 tumor suppressor activity and mutant p53 gain-of-function on cell growth. Gene 2001;277:15-30.

22 Al-Bozom IA. p53 and Bcl-2 oncoprotein expression in placentas with hydropic changes and partial and complete moles. APMIS 2000;108:756-60.

23 Chen CA, Chen YH, Chen TM et al. Infrequent mutation in tumor suppressor gene p53 in gestational trophoblastic neoplasia. Carcinogenesis 1994;15:2221-3.

24 Cheung AN, Shen DH, Khoo US et al. Immunohistochemical and mutational analysis of p53 tumor suppressor gene in gestational trophoblastic disease: correlation with mdm2, proliferation index, and clinicopathologic parameters. Int J Gynecol Cancer 1999;9:123-30.

25 Cheung AN, Srivastava G, Chung LP et al. Expression of the p53 gene in trophoblastic cells in hydatidiform moles and normal human placentas. J Reprod Med 1994;39:223-7.

26 Fulop V, Mok SC, Genest DR, Gati I, Doszpod J., Berkowitz RS. p53, p21, Rb and mdm2 oncoproteins. Expression in normal placenta, partial and complete mole, and choriocarcinoma. J Reprod Med 1998;43:119-27.

27 Halperin R, Peller S, Sandbank J, Bukovsky I, Schneider D. Expression of the p53 gene and apoptosis in gestational trophoblastic disease. Placenta 2000;21:58-62.

28 Uzunlar AK, Yilmaz F, Bayhan G, Akkus Z. Expressions of p53, proliferating cell nuclear antigen, and $\mathrm{Ki}-67$ in gestational trophoblastic diseases. Eur J Gynaecol Oncol 2002;23:79-83.

$29 \mathrm{Wu} \mathrm{Y}$, Zhang Q, Tang H. [Detection of p53 gene mutations in human gestational trophoblastic neoplasia by polymerase chain reaction-single strand conformational polymorphism techniques] [Chinese]. Hunan Yi Ke Da Xue Xue Bao 1997;22:338-40.

30 Persaud V, Ganjei P, Nadji M. Cell proliferative activity and mutation of P53 suppressor gene in human gestational trophoblastic disease. West Indian Med J 1993;42:142-3.

31 Shi YF, Xie X, Zhao CL et al. Lack of mutation in tumour-suppressor gene p53 in gestational trophoblastic tumours. $\mathrm{Br} J$ Cancer 1996;73:1216-9.

32 Rogel A, Popliker M, Webb CG., Oren M. p53 cellular tumor antigen: analysis of mRNA levels in normal adult tissues, embryos, and tumors. Mol Cell Biol 1985;5:2851-5.

33 Lane DP., Benchimol S. p53: oncogene or anti-oncogene?. Genes Dev 1990;4:1-8.

34 Webley KM, Shorthouse AJ, Royds JA. Effect of mutation and conformation on the function of p53 in colorectal cancer. J Pathol 2000;191:361-7.

Accepted for publication February 24, 2006 